\title{
A Commentary on Axon guidance pathway genes and Parkinson's disease
}

\author{
Hiroyuki Tomiyama
}

Journal of Human Genetics (2011) 56, 102-103; doi:10.1038/jhg.2010.153; published online 2 December 2010

\begin{abstract}
A xon guidance pathway is one of the $A_{\text {critical processes related to connectivity }}$ and repair of the wiring of the brain during the central nervous system development and throughout the lifetime in humans. Indeed, many axon guidance molecules have been shown to persist in the central nervous system having roles in not only precise neuronal network formation during development but also maintenance and plasticity of neural circuits. $^{1}$
\end{abstract}

In 1997, Livesey et al. ${ }^{2}$ reported that genetic variability in the axon guidance pathway was a possible factor contributing to the development of one of the major neurodegenerative disorders, Parkinson's disease (PD), in Caucasians. In 2005, genome-wide association study by Maraganore et al. ${ }^{3}$ revealed variants (single-nucleotide polymorphisms (SNPs)) in the axon guidance pathway genes related to PD susceptibility. The five mapped axon guidance pathway genes-deleted in colorectal carcinoma (DCC), ephrin receptor B1 (EPHB1), netrin-G1 (NTNG1), semaphorin 5A (SEMA5A) and SLIT3 - were found to have SNPs associated with the prediction of PD outcomes (susceptibility, survival free of PD and age at onset of PD). ${ }^{3,4}$ Especially, SEMA5A had significant association with $\mathrm{PD}$, suggesting a possible role for axon guidance in the pathogenesis of PD, such as early neurodegeneration in the subclinical period and possibly even during brain development (the miswiring hypothesis). ${ }^{3,4}$

Functionally, the axon guidance pathway consists of four major classes of ligands (ephrin, netrin, semaphorin and slit pro-

Dr $\mathrm{H}$ Tomiyama is at the Department of Neurology, Juntendo University School of Medicine, 2-1-1 Hongo, Bunkyo, Tokyo 113-8421, Japan.

E-mail: tomiyama@juntendo.ac.jp teins), their respective receptors (for example, eph, DCC and unc, neuropilin and plexin and robo proteins) and several downstream signaling proteins. ${ }^{4}$ DCC-deficient adult mice showed altered dopamine transmission and locomotor activity accompanied by reduced dendritic spine density in the cerebral cortex. ${ }^{5}$ These findings show that DCC is a crucial molecule in the development of dopamine circuitry, and alterations in DCC levels can lead to cognitive and behavioral abnormalities in adulthood. ${ }^{4,5}$ In EPHB1-knockout mice, there is a significant cell loss in the substance nigra pars reticulata, but there is no obvious change in the number of dopamine neurons in the substance nigra pars compacta. ${ }^{6}$ Differential expression of NTNG1 could alter dopaminergic and glutamatergic circuitry. ${ }^{4}$ SEMA5A could be involved in increasing the risk of PD by causing synaptic activity dysfunction and inflammation. ${ }^{4}$ Concerning SLIT3, Slit3/robo pathway is thought to initiate and accelerate the processes or progression of $\mathrm{PD}^{4}$ Thus, several axon guidance pathway genes and proteins have been considered as important molecules for dopamine axonal maintenance, regeneration and target recognition.

However, some subsequent studies, including analyses on other genes, showed that the axon guidance pathway genes had no association or very weak association with PD. Although one replication study did not replicate the data, two other replication studies performed pathway-based analyses of three genome-wide association data sets of $\mathrm{PD}$ and concluded that axon guidance was significantly associated with PD susceptibility. ${ }^{7,8}$ The discrepancy complicated our understandings of the role of the axon guidance pathway in PD. Although disease associations for single SNPs having small effects are difficult to replicate, the findings of the axon guidance pathway genes and the 'common disease-multiple rare variants' hypothesis suggest that the combined effects of variants within functionally related genes in certain pathways could have significant or larger effects.

In this issue, Kim et al. ${ }^{9}$ investigated whether the genetic variability in the axon guidance pathway is a susceptibility factor in PD patients in the Korean population. To my knowledge, this is the first report from an Asian population about variants in the axon guidance pathway genes in PD. A set of 22 SNPs was analyzed in 373 patients and 384 healthy controls, and the risk of PD was evaluated using odds ratios in unconditional and conditional logistic regression models in age- and gender-matched subsets. Among the axon guidance pathway genes, DCC rs17468382 and EPHB1 rs2030737 SNPs were found to be significantly associated with increased PD risk, and the CHP rs6492998 and RRAS2 rs2970332 SNPs were found to be significantly associated with reduced PD risk. They found no association of SEMA5A, which was thought to have a role in the development of dopaminergic neurons and to interact with other proteins in the axon guidance pathway, with PD. Subsequent multidimensionality reduction analysis to explore potential gene-gene interactions showed that there were no significant interactions among the above-mentioned SNPs.

Although two recent large genome-wide association study studies in Japanese and Europeans have not reported the involvement of the axon guidance pathway genes in $\mathrm{PD},{ }^{10,11}$ the study in the Korean population provides new data suggesting that these genes were susceptibility factors in PD patients; this made us reconsider the role of the axon guidance pathway in the pathogenesis of 
PD. Although no significant interactions among their SNPs in the axon guidance pathway genes were observed, investigations on interactions among other common and rare variants in the key genes of $\mathrm{PD}$, such as $\alpha$-synuclein and LRRK2, could be interesting. Thus, larger studies on candidate genes and SNPs or further genome-wide association study data analyses in various populations are warranted. Even if each variant has only a weak role, together added-up roles of many variants and gene-gene interaction of many genes in some common pathways could have a greater impact on the pathogenesis of multifactorial diseases such as PD.

Moreover, several lines of recent evidence support the challenging hypothesis that aberrant expression or function of axon guidance proteins such as semaphorins, ephrins, netrins and slits, which are normally involved in sculpting and maintaining motor neuron circuits, may induce pathological changes in the motor neuron circuitry and contribute to the pathogenic mechanism involved in the development of amyotrophic lateral sclerosis. ${ }^{12}$ Furthermore, SNP models for PD were subsequently refined and compared with those of the axon guidance pathway that were highly predictive of amyotrophic lateral sclerosis susceptibility. ${ }^{13}$
Therefore, the axon guidance pathway SNP models could be therapeutic targets (for example, axon-regeneration therapy) for neurodegenerative disorders such as PD and amyotrophic lateral sclerosis. The genomic pathway approach might lead to new breakthroughs in the multiple disease pathways for many common, complex or overlapping disorders.

Thus, the role of axon guidance pathway should be further elucidated in common pathways for the development of PD and other neurodegenerative disorders.

\section{CONFLICT OF INTEREST}

The author declares no conflict of interest.

1 Curinga, G. \& Smith, G. M. Molecular/genetic manipulation of extrinsic axon guidance factors for CNS repair and regeneration. Exp. Neurol. 209, 333-342 (2008).

2 Livesey, F. J. \& Hunt, S. P. Netrin and netrin receptor expression in the embryonic mammalian nervous system suggests roles in retinal, striatal, nigral, and cerebellar development. Mol. Cell Neurosci. 8, 417-429 (1997).

3 Maraganore, D. M., de Andrade, M., Lesnick, T. G., Strain, K. J., Farrer, M. J., Rocca, W. A. et al. Highresolution whole-genome association study of Parkinson disease. Am. J. Hum. Genet. 77, 685-693 (2005).

4 Lin, L., Lesnick, T. G., Maraganore, D. M. \& Isacson, O. Axon guidance and synaptic maintenance: preclinical markers for neurodegenerative disease and therapeutics. Trends. Neurosci. 32, 142-149 (2009).

5 Grant, A., Hoops, D., Labelle-Dumais, C., Prévost, M., Rajabi, H., Kolb, B. et al. Netrin-1 receptor-deficient mice show enhanced mesocortical dopamine transmission and blunted behavioural responses to amphetamine. Eur. J. Neurosci. 26, 3215-3228 (2007).

6 Richards, A. B., Scheel, T. A., Wang, K., Henkemeyer, M. \& Kromer, L. F. EphB1 null mice exhibit neuronal loss in substantia nigra pars reticulata and spontaneous locomotor hyperactivity. Eur. J. Neurosci. 25, 2619-2628 (2007).

7 Wang, K., Li, M. \& Bucan, M. Pathway-based approaches for analysis of genomewide association studies. Am. J. Hum. Genet. 81, 1278-1283 (2007).

8 Srinivasan, B. S., Doostzadeh, J., Absalan, F., Mohandessi, S., Jalili, R., Bigdeli, S. et al. Whole genome survey of coding SNPs reveals a reproducible pathway determinant of Parkinson disease. Hum. Mutat. 30, 228-238 (2008).

9 Kim, J.- M., Park, S. K., Yang, J. J., Shin, E.- S., Lee, J.- Y., Yun, J. Y. et al. SNPs in axon guidance pathway genes and susceptibility for Parkinson's disease in the Korean population. J. Hum. Genet. 56, 125-129 (2011).

10 Satake, W., Nakabayashi, Y., Mizuta, I., Hirota, Y., Ito, C., Kubo, M. et al. Genome-wide association study identifies common variants at four loci as genetic risk factors for Parkinson's disease. Nat. Genet. 41, 1303-1307 (2009).

11 Simón-Sánchez, J., Schulte, C., Bras, J. M., Sharma, M., Gibbs, J. R., Berg, D. et al. Genome-wide association study reveals genetic risk underlying Parkinson's disease. Nat. Genet. 41, 1308-1312 (2009).

12 Schmidt, E. R., Pasterkamp, R. J. \& van den Berg, L. $\mathrm{H}$. Axon guidance proteins: novel therapeutic targets for ALS? Prog. Neurobiol. 88, 286-301 (2009).

13 Lesnick, T. G., Sorenson, E. J., Ahlskog, J. E., Henley, J. R., Shehadeh, L., Papapetropoulos, S. et al. Beyond Parkinson disease: amyotrophic lateral sclerosis and the axon guidance pathway. PLoS One 3, e1449 (2008). 